\title{
The effect of radiometer placement and view on inferred directional and hemispheric radiometric temperatures of a urban canopy
}

\author{
C. Adderley et al.
}

Correspondence to: A. Christen (andreas.christen@ubc.ca) 


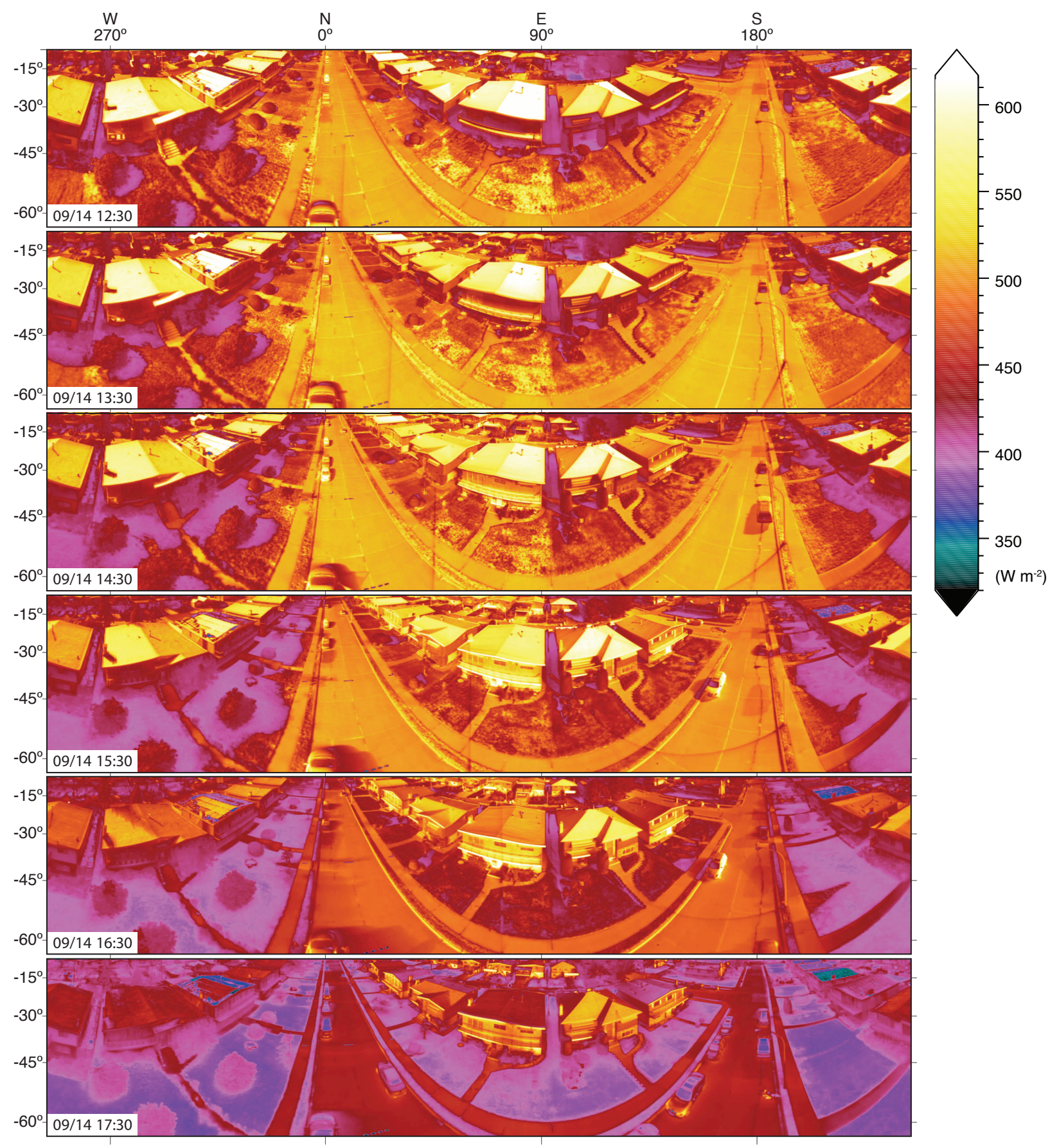

Figure S1 - Panoramic time sequential thermography (PTST) dataset from September 14, 2008, 12:30 to 17:30. Each panorama is composed of $\approx 120$ single images and projected using a conformal Mercator grid relative to the local horizon. 


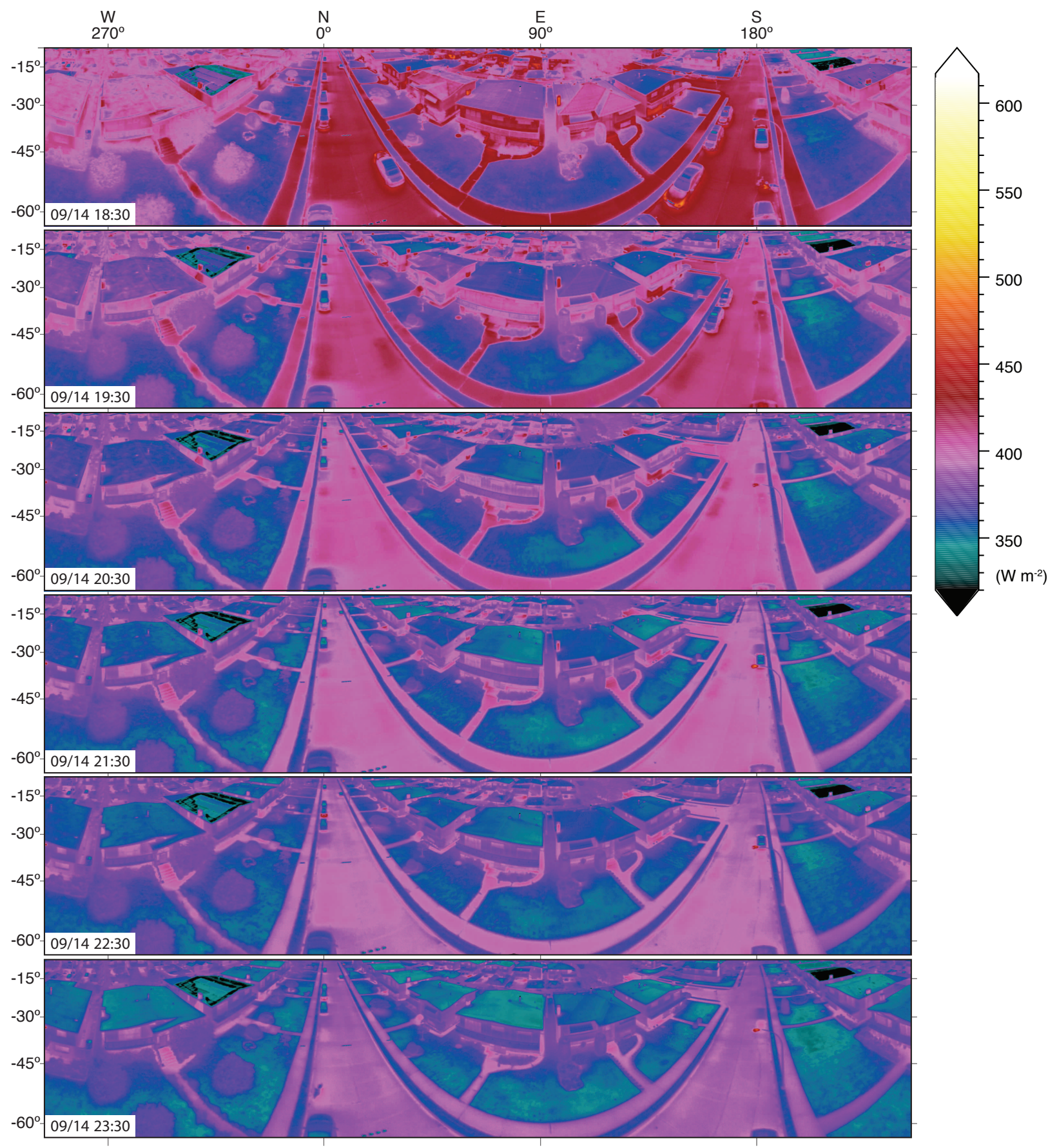

Figure S2 - Panoramic time sequential thermography (PTST) dataset from September 14, 2008, 18:30 to 23:30. Each panorama is composed of $\approx 120$ single images and projected using a conformal Mercator grid relative to the local horizon. 


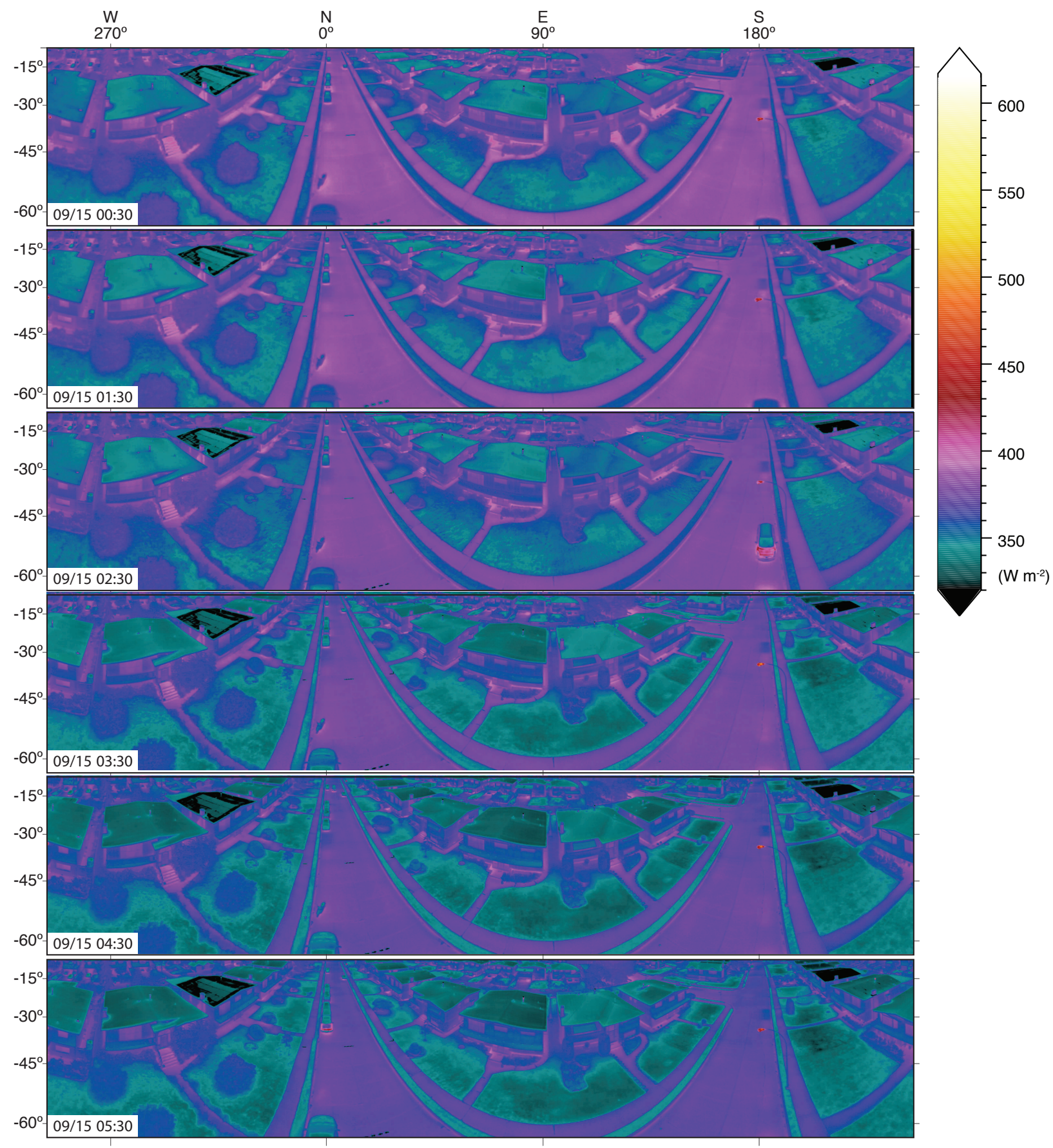

Figure S3 - Panoramic time sequential thermography (PTST) dataset from September 15, 2008, 00:30 to 05:30. Each panorama is composed of $\approx 120$ single images and projected using a conformal Mercator grid relative to the local horizon. 


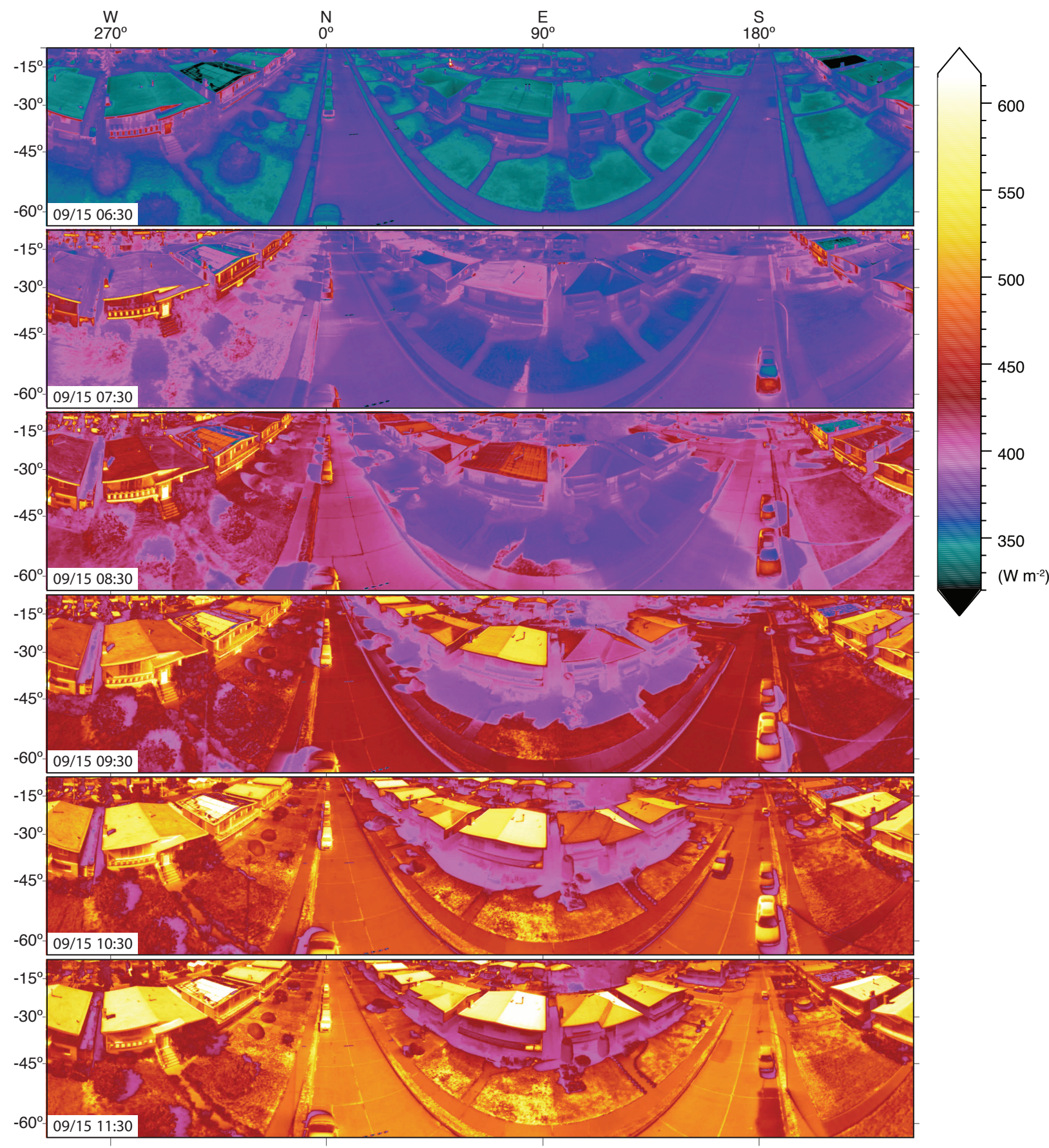

Figure S4 - Panoramic time sequential thermography (PTST) dataset from September 15, 2008, 06:30 to 11:30. Each panorama is composed of $\approx 120$ single images and projected using a conformal Mercator grid relative to the local horizon. 

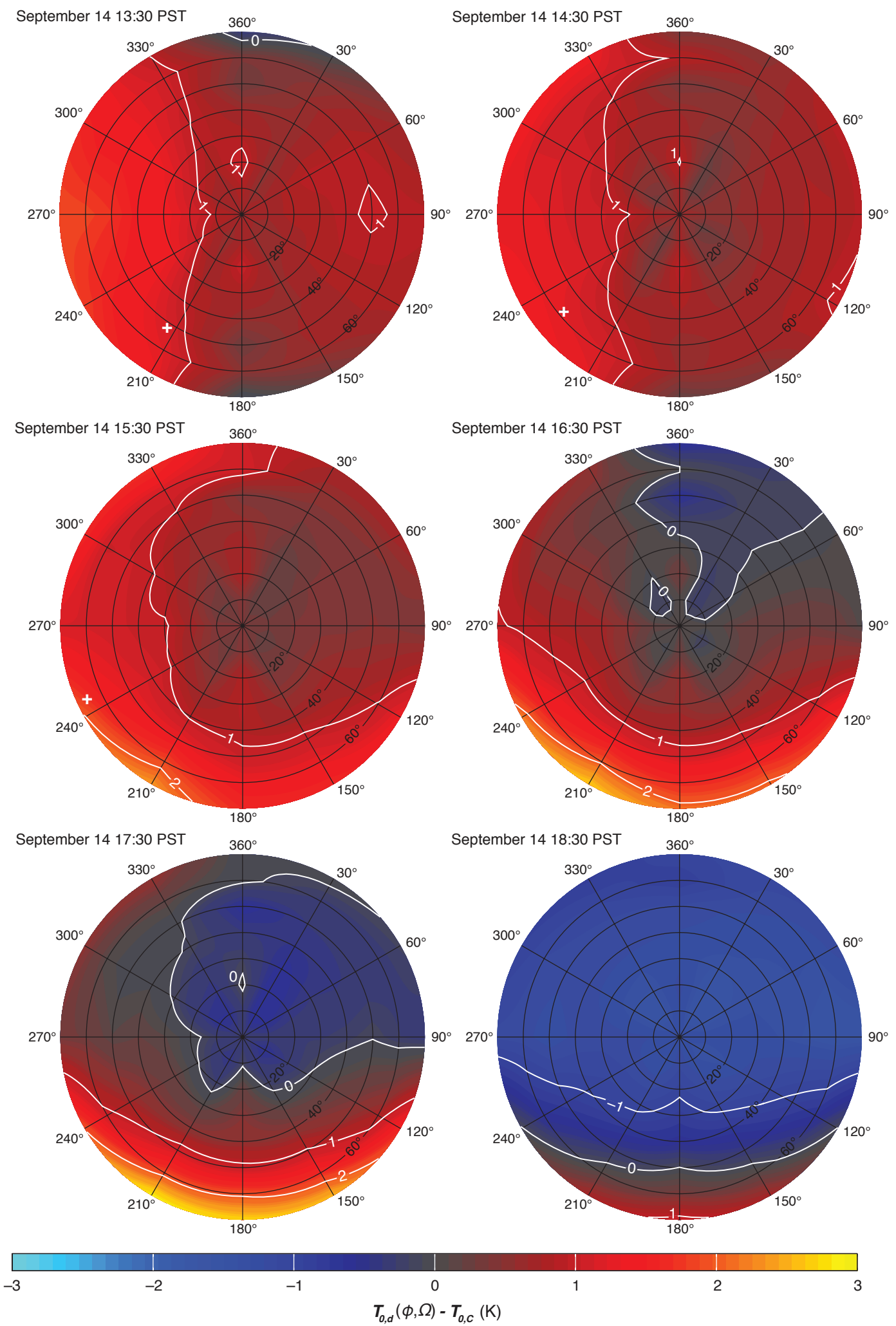

Figure S5 - The bias $T_{0, d}-T_{0, C}$ for sensors with a narrow IFOV in the nadir and various oblique angles similar to Figure 8 in journal article but for September 14 13:30 to September 14 18:30. 

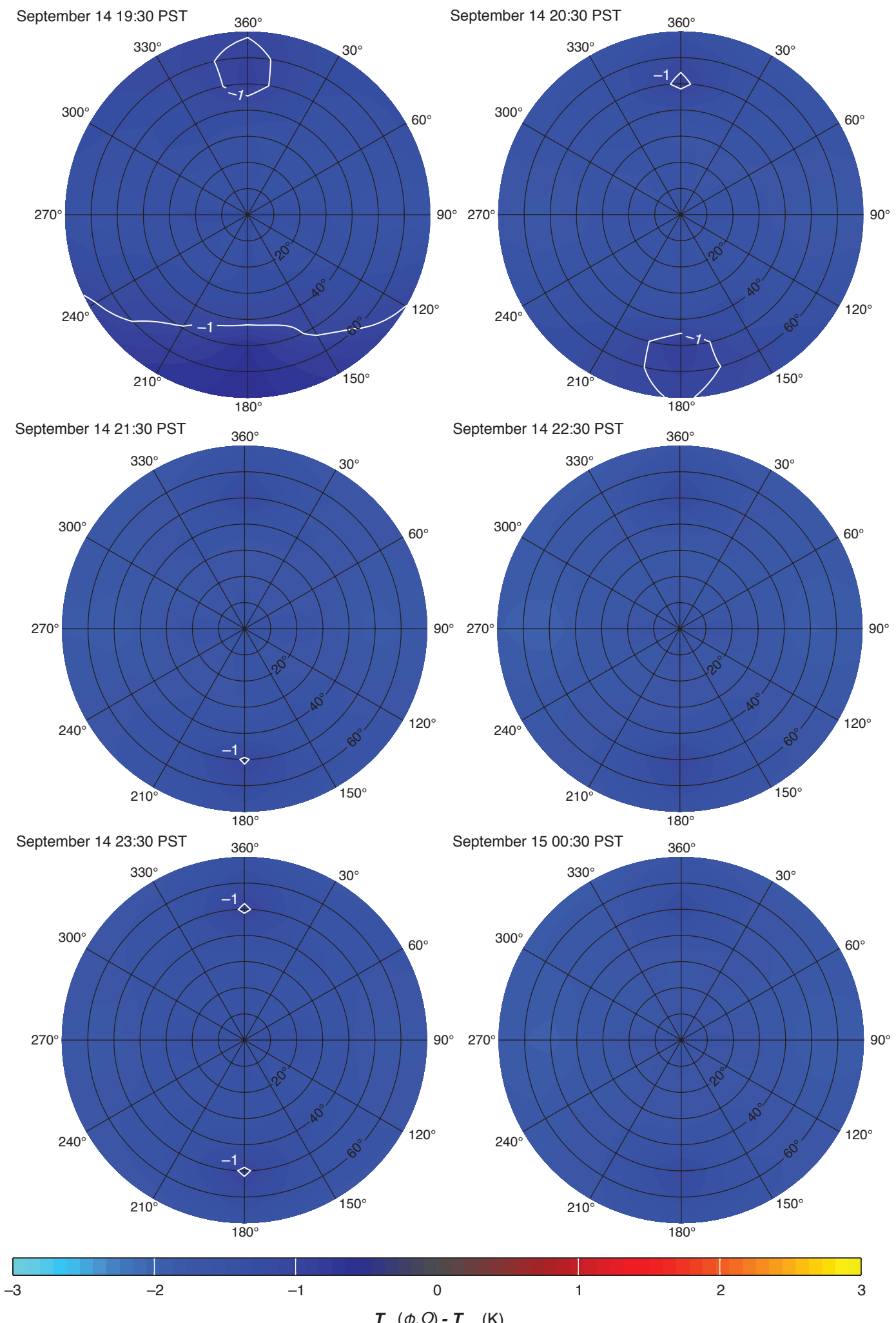

$T_{o, d}(\phi, \Omega)-T_{o, c}(\mathrm{~K})$

Figure S6 -The bias $T_{0, d}-T_{0, C}$ for sensors with a narrow IFOV in the nadir and various oblique angles similar to Figure 8 in journal article but for September 14 19:30 to September 15 00:30 

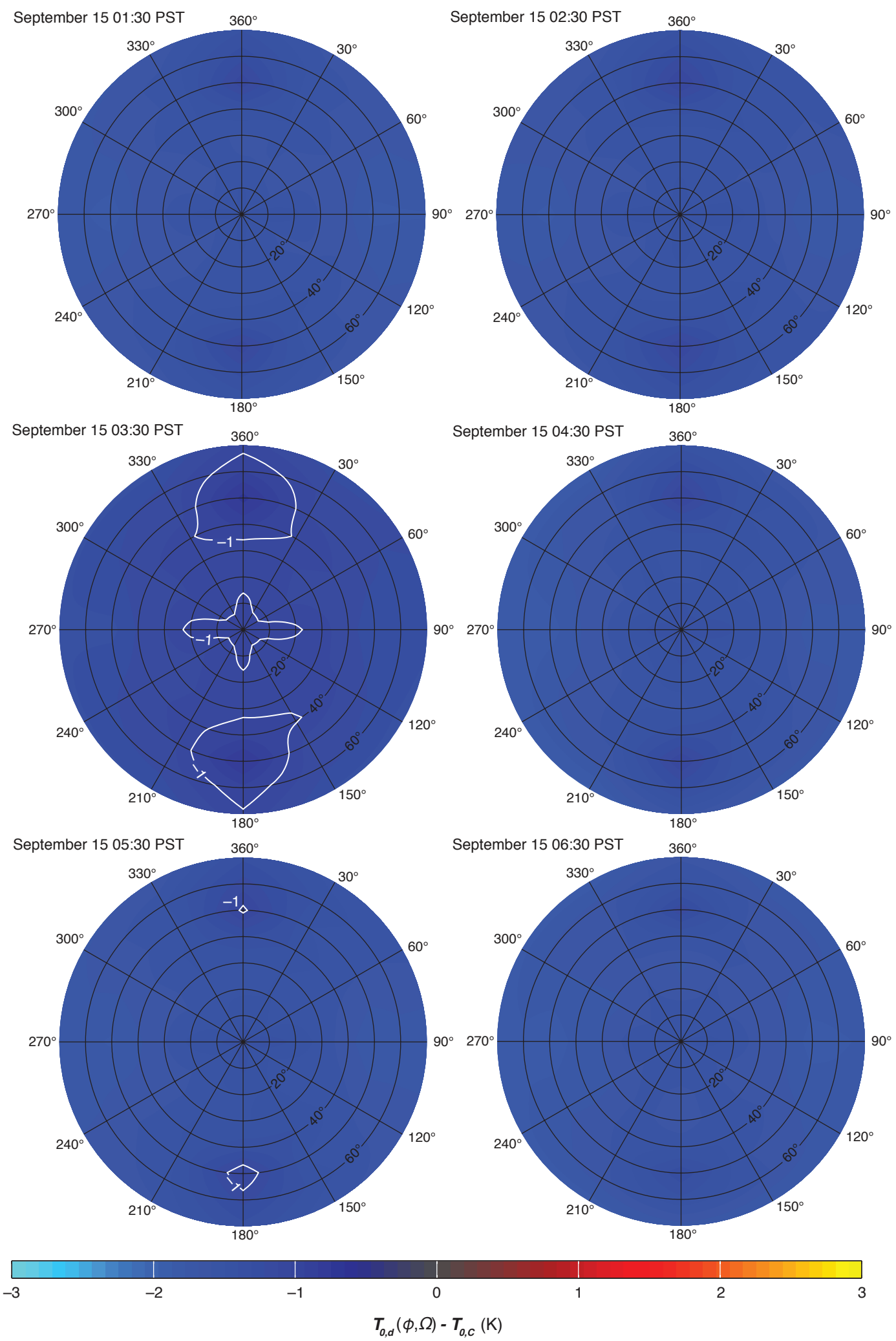

Figure S7 - The bias $T_{0, d}-T_{0, C}$ for sensors with a narrow IFOV in the nadir and various oblique angles similar to Figure 8 in journal article but for September 15 01:30 to September 15 06:30 

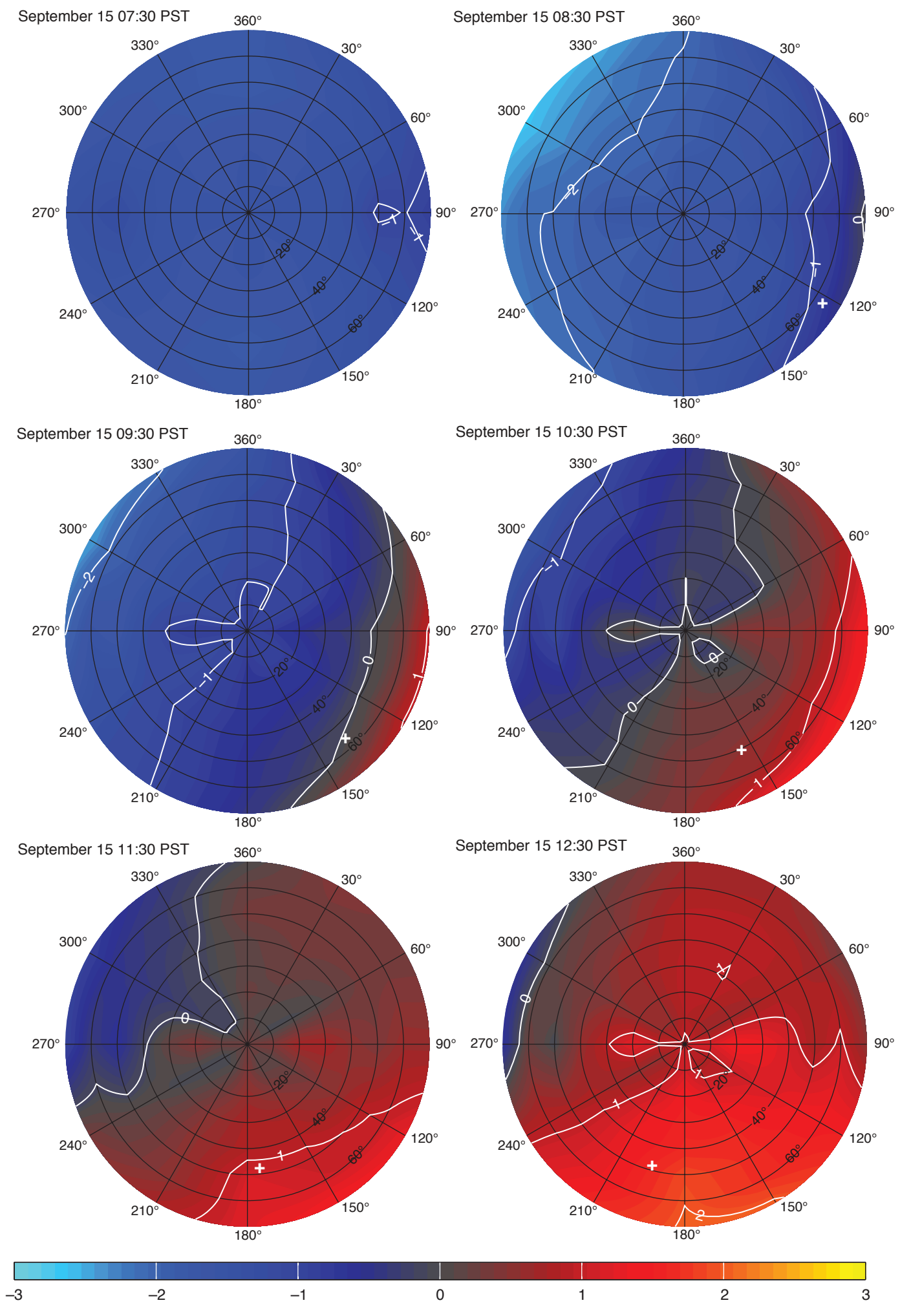

$T_{o, d}(\phi, \Omega)-T_{o, c}(\mathrm{~K})$

Figure S8 - The bias $T_{0, d}-T_{0, C}$ for sensors with a narrow IFOV in the nadir and various oblique angles similar to Figure 8 in journal article but for September 15 07:30 to September 15 12:30. 